\title{
An Analytical Simulation of Boundary Roughness for Incompressible Viscous Flows
}

\author{
JOHN VENETIS \\ Department of Applied Mathematics and Physical Sciences \\ National Technical University of Athens, \\ 5 Heroes of Polytechnion Avenue, Athens \\ GREECE
}

\begin{abstract}
The intention of this paper is to investigate the boundary roughness of a mounted obstacle which is inserted into an incompressible, external and viscous flow field of a Newtonian fluid. In particular, the present study focuses on the cross - sectional area of the obstacle, which is assumed to be a non deformable body (rigid object) with a predefined shape of random roughness. For facility reasons and without violating the generality, one may select the cross - section of the body which contains its center of gravity and is perpendicular to the main flow direction. The boundary of this cross - sectional area is mathematically simulated as the polygonal path of the length of a single - valued continuous function. Evidently, this function should be of bounded variation. The novelty of this work is that the formulation of the random roughness of the boundary has been carried out in a deterministic manner.
\end{abstract}

Key-Words: - Newtonian fluid, incompressible flow, mounted obstacle, random roughness, polygonal path, Riemann - Stieltjes integral

Received: September 26, 2020. Revised: February 20, 2021. Accepted: March 4, 2021.

Published: March 12, 2021.

\section{Introduction}

In the current literature, the irregular boundaries are mostly modeled as spatially homogenous random processes. However, the spatial variations are many times very small for computational grids [1]. Nonetheless, the majority of geometric models which currently appear in literature and are based on an Analytical or Differential Geometry viewpoint, have not included any particular deterministic formulation for the roughness of the circumstantial boundaries $[2,3,4,5]$. On the other hand, referring to the influence of the roughness of an arbitrary surface, one may report that any surface which is inserted into an external viscous flow is called "smooth", when its roughness is less than the averaging thickness of the viscous sub layer, which is evidently at least the $1 \%$ of the thickness of the self - preserving turbulent boundary layer [6,7]. In the meanwhile, during conceptual design process of aircrafts or some special architectural forms, the rigid body can be mathematically simulated by creating a stack of cross sections and then lofting in between these sections to create a smooth shape $[8,9,10]$. In such cases, the boundaries of these cross sectional areas are not self - intersecting or continuously constructing curves [11]. In the past years, there is a lot of recent research work carried out for the investigation of boundary roughness concerning mounted and/or rotated obstacles. In Ref. [12] a unified approach to time periodic incompressible viscous fluid flow problems is presented. Besides, a considerable study on the influence of surface roughness on shear viscosity was performed in Ref. [13]. Moreover, in Ref. [14] a comprehensive investigation into how the boundary roughness affects a fluid flow through a corrugated pipe is presented, whereas for a detailed study on the influence of surface roughness on a separating turbulent boundary layer one may refer to Ref [15]. Further, in Ref. [16] an experimental study of rotating - disk boundary layer flow with surface roughness was carried out. In this context, it was shown that if the surface is sufficiently rough, laminar-turbulent transition can occur via a convectively unstable route ahead of the onset of absolute instability. Finally, in Ref. [17] an applicable investigation on the influence of rotating wheels, ride height and wheelhouse geometry on the drag coefficient of electric vehicle was carried out 
by means of Computational Fluid Dynamics (CFD) techniques. The objective of the present work aims at examining the roughness of the boundary of a mounted obstacle inserted for an incompressible viscous flow field. The mathematical formalism that we shall develop here in order to carry out a deterministic approximation of the roughness of a two - dimensional boundary, premises beforehand two fundamental assumptions which concern the geometrical features of this boundary. In particular, the rough boundary of the cross sectional area of the mounted obstacle, due to its random roughness, has not been considered as:

i) Piece - wise smooth and parameterized surface from Differential Geometry standpoint.

ii) Smooth surface from Fluid Dynamics standpoint.

In this framework, the boundary has been simulated as the polygonal path of the length of a single valued continuous function. Evidently, this function should be assumed beforehand to be of bounded variation. The novelty of this work is that the formulation of the random roughness of the boundary has been carried out by means of a deterministic method and thus does not involve stochastic random processes.

\section{Problem Formulation}

\subsection{Towards an analytical simulation of boundary geometry}

It is known that for any type of external viscous flow patterns, along the boundary, which marginally constitutes a stream surface, the following statement holds $[6,18]$

$$
\begin{aligned}
& V_{x}=V_{y}=V_{z}=0 \Rightarrow \\
& V_{x}+V_{y}+V_{z}=0
\end{aligned}
$$

Thus, taking into consideration the well-known relationship which describes the network of the streamlines according to Eulerian formalism for the description of a flow field $[18,19]$, one may obtain the following relationship:

$$
\frac{d x}{V_{x}}=\frac{d y}{V_{y}}=\frac{d z}{V_{z}}=\frac{d x+d y+d z}{V_{x}+V_{y}+V_{z}}
$$

Eqns. (1) and (2) can be combined to yield $x+y+z=c t$

Moreover, according to eqn. (2) one may deduce that

$$
\begin{aligned}
& x d x+y d y+z d z=c t \Leftrightarrow \\
& \frac{1}{2} d x^{2}+\frac{1}{2} d y^{2}+\frac{1}{2} d z^{2}=c t \Leftrightarrow \\
& x^{2}+y^{2}+z^{2}=Q
\end{aligned}
$$

where $Q \in R_{+}^{*}$

Thus, given a specific value of the parameter $Q$, one may also derive the equation of the corresponding osculating sphere for an arbitrary point lying on the boundary of the mounted obstacle.

In the meanwhile, it is known that any two dimensional curve the tangent lines of which have geometric properties independent of the circumstantial contact point, leads to an Ordinary Differential Equation (ODE) of Clairaut type [20, 21]. It is well - known that the complete solution of this ODE constitutes a single - parameter bundle of lines [20,21]. Obviously, these lines have the same geometric features with the tangent lines of the aforementioned curve. In this context, one may also deduce that the envelope of this bundle of lines coincides with any level curve of the surface - area of the mounded obstacle for each seperate value of the parameter. In a motionless rectangular Cartesian frame of reference, the coordinates of any tangent line are given as: $\left(x-\frac{y}{y^{\prime}}, y-x y^{\prime}\right)$

Hence the differential form of the curve is:

$$
\begin{aligned}
& x-\frac{y}{y^{\prime}}+y-x y^{\prime}=\kappa, \kappa \in R \Leftrightarrow \\
& x y^{\prime}-+y y^{\prime}-x\left(y^{\prime}\right)^{2}=\kappa y^{\prime} \Leftrightarrow \\
& y=x y^{\prime}+\frac{\kappa y^{\prime}}{y^{\prime}-1}
\end{aligned}
$$

where $y \neq x+c$ 
The complete solution of eqn. (5) is expressed in the following single - parameter representation [20, 21]:

$y=c x+\frac{\kappa c}{c-1}, c \neq 1 \Leftrightarrow$

$x c^{2}-(y+x-\kappa) c+y=0$

where $c \neq 1$

Apparently, eqn. (6) constitutes a single - parameter family of lines.

Consequently, the envelope of eqn. (6) can be estimated as

$$
\begin{aligned}
& (y+x-\kappa)^{2}-4 x y=0 \Leftrightarrow \\
& (y+x-\kappa)^{2}=4 x y \Leftrightarrow \\
& y+x-\kappa=2 \sqrt{x} \sqrt{y} \\
& \vee \\
& y+x-\kappa=-2 \sqrt{x} \sqrt{y} \\
& y+x-2 \sqrt{x} \sqrt{y}=\kappa \\
& \vee \\
& y+x+2 \sqrt{x} \sqrt{y}=\kappa
\end{aligned} \Leftrightarrow
$$$$
\sqrt{x}+\sqrt{y}=\sqrt{\kappa}
$$

$\checkmark$

$\sqrt{x}-\sqrt{y}=\sqrt{\kappa}$

Evidently, the mathematical disjunction consisting of eqns. (7) $\vee(8) \vee(19)$ is equivalent to eqn. (6).
Besides, eqns. (7), (8) and (9) can be solved for the variable $y$, resulting in the following mathematical disjunction:

$$
y=(\sqrt{\kappa}-\sqrt{x})^{2}
$$

$\vee$

$$
y=(\sqrt{\kappa}+\sqrt{x})^{2}
$$

\subsection{Towards an analytical formulation of the boundary roughness}

Suggestively, let us select eqn. (10) letting the variable $x$ lie over an arbitrary interval $[a, b]$.

Then we shall evaluate the polygonal approximation of the length of this function.

In this context, to formulate the roughness of any possible curve motivated by eqn. (10) from the differential point of view, we need to estimate the polygonal approximations of the lengths of these functions with respect to an arbitrary partition $p$ of the interval $[a, b]$ taking also into account that these functions are of bounded variation in their domain of definition [22]. Hence, we can write out:

$$
\begin{aligned}
& L(f, p)=\sum_{n=1}^{n}\left(\left(\lambda_{n}-\lambda_{n-1}\right)^{2}+\left(f\left(\lambda_{n}\right)-f\left(\lambda_{n-1}\right)\right)\right)^{1 / 2} \\
& \forall p=\left\{a=\lambda_{0}<\lambda_{1}<\ldots<\lambda_{n}=b\right\}, n \in N
\end{aligned}
$$

Apparently, the following mathematical conjunction holds:

$$
\min L(f, p)=V(f, p)
$$

$\wedge$

$$
\max L(f, p)=V(f, p)+(b-a)
$$

where

$$
\begin{aligned}
& V(f, p)=\sum_{n=1}^{n}\left|f\left(\lambda_{n}\right)-f\left(\lambda_{n-1}\right)\right| \\
& \text { with } p=\left\{a=\lambda_{0}<\lambda_{1}<\ldots<\lambda_{n}=b\right\}, n \in N
\end{aligned}
$$


Evidently when the cardinality of this partition increases, also the roughness of the boundary does.

On the other hand, it is also known $[22,23]$ that any finite summation can be represented equivalently as the Riemann - Stieltjes integral of an appropriate single - valued function, which cannot be obtained in a direct manner. In this context, one may proceed as follows:

Let $h:[0, \infty) \rightarrow R$ be a continuous single valued function.

Then for every interval:[0,x]:x $\geq 1$ the following relationship holds:

$$
\sum_{n=1}^{[x]} h(n)=\int_{0}^{x} h(t) d[t]
$$

Hence one may request the validity of the following equality:

$$
L(f, p)=\int_{0}^{x} h(t) d[t]
$$

On the other hand, it is known from Calculus that referring to the integer part of any real variable the following statements hold:

$$
\begin{aligned}
& 0 \leq t<1 \Leftrightarrow[t]=0 \\
& 1 \leq t<2 \Leftrightarrow[t]=1 \quad\left(18 \mathrm{~A}_{1}\right) \\
& 2 \leq t<3 \Leftrightarrow[t-2]=0 \quad\left(18 \mathrm{~A}_{2}\right) \\
& 3 \leq t<4 \Leftrightarrow[t-2]=1
\end{aligned}
$$

$$
\begin{aligned}
& {[\mathrm{x}]-2 \leq t<[\mathrm{x}]-1 \Leftrightarrow[\mathrm{t}-[\mathrm{x}]+1]=0 \quad\left(18 \mathrm{~A}_{\mathrm{x}-1}\right)} \\
& {[\mathrm{x}]-1 \leq \mathrm{t}<[\mathrm{x}] \Leftrightarrow[\mathrm{t}-[\mathrm{x}]+1]=1 \quad\left(18 \mathrm{~A}_{\mathrm{x}}\right)}
\end{aligned}
$$

Consequently, over the interval $[0,2)$ one may infer

$$
[t]=H(t)
$$

where $H(t)$ denotes the Heaviside step function, which is also known as the Unit Step Function.
Evidently the following relationship holds,

$$
[0,2] \subset[0,[x]]
$$

In this framework, one may proceed in the same manner respectively, over the next intervals $[[2,4], \ldots,[[\mathrm{x}]-2,[\mathrm{x}]]$.

Besides, by differentiating eqn. (19) withy respect to $t$, it implies that

$$
d[t]=d H(t)
$$

Next, since Heaviside function satisfies Dirichlet's principles, it can be expanded in a Fourier series as follows

$$
H(t)=\frac{1}{2}+\frac{2}{\pi} \sum_{k=1}^{+\infty} \frac{\sin (2 k+1) t}{2 k+1}
$$

Apparently, the above approximation holds over the interval $[-\pi,+\pi]$ and also at $\mathrm{t}=0$ and at $\mathrm{t}= \pm \pi$ the corresponding series converges to $\frac{1}{2}$.

Thus, one may deduce that this approximation holds if and only if $\mathrm{t}<\pi+[x]-1$

Nonetheless, the above restriction does not violate the proposed mathematical formalism to estimate the boundary roughness.

In continuing, a differentiation of eqn. (21) with respect to $t$ yields

$$
d H(t)=\frac{2}{\pi}(\cos t+\cos 3 t+\ldots+\cos ((2 k+1) t)) d t
$$

Eqn. (22) and eqn. (20) can be combined with to yield

$$
d[t]=\frac{2}{\pi}(\cos t+\cos 3 t+\ldots+\cos ((2 k+1) t)) d t
$$

\section{Problem Solution}

Now, for facility reasons one may additionally assume that the continuous function $h(t)$ which first appeared in eqn. (16) is analytical over its domain of definition, and therefore it can be expanded in Taylor series centred at any point of this domain. 
Hence, this aforementioned function can be represented by an Nth - degree polynomial $P(x)$ such that

$$
\begin{aligned}
& h(0)=P(0) \\
& \wedge \\
& h^{(n)}(0)=P^{(n)}(0) \\
& \forall n \in N^{*}
\end{aligned}
$$

In this framework, eqn. (17), eqn. (22) and eqn. (23) can be combined to yield

$$
L(f, p)=\frac{2}{\pi} \int_{0}^{x} h(t)\left(\begin{array}{l}
\cos t+\cos 3 t+ \\
+\ldots+\cos ((2 k+1) t)
\end{array}\right) d t
$$

In continuing, to calculate the integral on the right hand side of eqn. (24) one may take into consideration that the antiderivative

$$
\int \cos (A t) P(t) d x, \forall A \in R^{*} \text { is given as }
$$

$$
\int \cos (A t) P(t) d t=\frac{1}{A} \cdot\left[\begin{array}{l}
\sin (A \cdot t) P(t)+ \\
+\frac{1}{A^{1}} \cdot P^{(1)}(t) \cdot \sin \left(A \cdot t+1 \cdot \frac{\pi}{2}\right)+ \\
+\frac{1}{A^{2}} \cdot P^{(2)}(t) \cdot \sin \left(A \cdot t+2 \cdot \frac{\pi}{2}\right)+\ldots \\
\ldots+\frac{1}{A^{n}} \cdot P^{(n)}(t) \sin \left(A \cdot t+n \cdot \frac{\pi}{2}\right)
\end{array}\right]+C_{1}
$$

Since $P^{(n+1)}(t) \equiv 0$

Thus we can write out

$$
L(f, p)=\left[\sum_{j=1}^{n} \sum_{k=1}^{m} \frac{P^{(j)}(x)}{(2 k+1)^{j}} \sin \left((2 k+1)+\frac{j \cdot \pi}{2}\right)\right]_{0}^{x}
$$

Then, after the necessary algebraic manipulations eqn. (26) finally yields

$$
\begin{aligned}
L(f, p)= & \sum_{j=1}^{n} \sum_{k=1}^{m} \frac{P^{(j)}(x)}{(2 k+1)^{j}} \sin \left((2 k+1)+\frac{j \cdot \pi}{2}\right) \\
& -\sum_{j=1}^{n} \sum_{k=1}^{m} \frac{P^{(j)}(0)}{(2 k+1)^{j}} \cdot(-1)^{\frac{j-1}{2}}
\end{aligned}
$$

Summarizing, one may infer that should the single valued continuous functions introduced by eqns.
(10) and (11) be replaced by their corresponding Taylor expansions, the "roughness" of their graphs drawn in a rectangular Cartesian frame of reference, can be reliably simulated in a deterministic manner as the polygonal approximation of the length $\mathrm{L}$ ( $\mathrm{f}$, p).

\section{Discussion}

In the previous unit, a deterministic mathematical formulation for the roughness of a boundary of a mounted obstacle, which is inserted into an external incompressible viscous flow of a Newtonian fluid was performed. To accommodate our mathematical analysis, we centred on the cross - sectional area of the obstacle which contains its centroidal axis and is perpendicular to the main flow direction. The boundary roughness of the obstacle was modeled as the polygonal approximation of the length of a function which was assumed beforehand to be of bounded variation. This method could be further utilized and/or developed, by its implementation in parallel with Johnson's mathematical formalism [24, 25, 26], which concerns convex polyhedra with regular faces, since all convex functions satisfy Lipschitz condition and indeed are of bounded variation. In this context, one may take into account that the envelope of the cross - sectional area of any convex polyhedron constitutes the graph of a Lipschitz function. Thus, the cross - sectional area of any convex polyhedron, could be considered as the polygonal approximation of the length of a single - valued convex function. In addition, one may say that this proposed method could be improved and refined by taking into account some exact and / or approximate forms of the unit step function presented in Refs.[27, 28, 29]

\section{Conclusion}

The aim of this paper was to present a deterministic mathematical formulation for the boundary roughness of a mounted obstacle, which is inserted into an external viscous flow of a Newtonian fluid. In this context, the author considered the cross sectional area of the obstacle which contains its center of gravity and also is perpendicular to the main flow direction. This area was assumed to have a predefined shape but random roughness. Then by means of a Mathematical Analysis viewpoint, the boundary roughness of this area was simulated as the polygonal approximation of the length of a continuous function. This function was assumed to 
be of bounded variation. The novelty of this work is that the introduced mathematical model is deterministic throughout and does not involve stochastic random processes. This method could be further exploited and utilized by its implementation together with Johnson's theory for convex polyhedra with regular faces, since every convex function satisfies Lipschitz condition and evidently is of bounded variation.

\section{References:}

[1] Gerard-Varet D. The Navier Wall Law at a Boundary with Random Roughnes, Communications in Mathematical Physics Vol. 286, No. 1, 2009, pp. 81-110

[2] Ball W.W., Coxeter H.S. Mathematical recreations and essays 13th ed. Dover New York, 1987

[3] Cundy H.M., Rollet A.P. Mathematical Models, Third ed. Tarquin, Stradbroke, 1981

[4] Fejes Toth. L. Regular Figures Pergamon, Press Oxford 1964

[5] Ferguson, J. Multivariable curve interpolation. Journal of the Association for Computing Machinery, Vol. 11, No. 2, 1964, pp. 221-228.

[6] Schlichting H. Boundary Layer Theory, 7th Edition, Mc Graw Hill, New York, 1979

[7] Wu J.C. Problems of general viscous fluid flow (Chapters I,II) Elsevier Applied Science Publ. London and New York 1982

[8] Liming, R.A., Conic Lofting of Streamline Bodies: The Basic Theory of a Phase of Analytic Geometry Applicable to Aircraft, Aircraft Engineering and Aerospace Technology, Vol. 19, No. 7, 1947, pp. 222-228.

[9] Strud I. Boundary Representation Modelling Techniques, Springer London 2006

[10] Bezier P. General Distortion of an ensemble of biparametric surfaces. Computer-Aided Design Vol. 10, No. 2, 1977, pp. 116-20.

[11] Blinn, J. F., A Generalization of Algebraic Surface Drawing, Association for Computing Machinery Transactions on Graphics Vol. 1, No. 3, 1982, pp. 235-256.
[12] Geissert, M., Hieber, M. and Nguyen, T.H., A General Approach to Time Periodic Incompressible Viscous Fluid Flow Problems, Archive for Rational Mechanics and Analysis Vol. 220, No.1, 2016, pp. 1095-1118

[13] Michail Papanikolaou, Michael Frank, and Dimitris Drikakis, Effects of surface roughness on shear viscosity, Physical Review E Vol 95, No. 3, 2017, ID 033108

[14] Eduard Marušić-Paloka, Igor Pažanin, Effects of boundary roughness and inertia on the fluid flow through a corrugated pipe and the formula for the Darcy-Weisbach friction coefficient, International Journal of Engineering Science, Volume 152, No. 1, 2020 ID 103293

[15] Wu, W. and Piomelli, U. Effects of surface roughness on a separating turbulent boundary layer, Journal of Fluid Mechanics Vol. 841, No. 1 2018, No. 4, pp. 552-580.

[16] Imayama, S., Alfredsson, P. H. andd Lingwood, R. J. Experimental study of rotating disk boundary-layer flow with surface roughness. Journal of Fluid Mechanics Vol. 786, No. 1, 2016, pp. 5-28.

[17] A. Alekseev, A. Maksimov, A. Tarasov, CFD Investigations of the Effect of Rotating Wheels, Ride Height and Wheelhouse Geometry on the Drag Coefficient of Electric Vehicle, International Journal of Mechanics, NAUN, pp. 130-134, Volume 14, 2020

[18] Ladyzhenskaya. O.A. The mathematical theory of viscous incompressible flow,

Gordon and Breach Science Publishers New York 1969 (reprint 1975)

[19] Lions P.L. Mathematical topics in fluid mechanics Vol. 1 Incompressible models Oxford Lecture Series in Mathematics and its Applications 3, Oxford University Press, 1996

[20] Krasnov M. Ordinary Differential Equations MIR Publications Moskow, 1983

[21] Tikhonov A., Vasileva A., Sveshnikov A. Differential Equations, Springer - Verlag, 1980 
[22] Segel L.A. Mathematics Applied to Continuum Mechanics, Dover, 1977

[23] Arfken G. Mathematical Methods for Physicists Second edition Academic Press New York 1970

[24] Coxeter H.S. Projective Geometry 2nd ed. Springer - Verlag 1974

[25] Johnson N. W. Convex Polyhedra with Regular Faces Canada J. Math. 18169 - 2001966

[26] A. Pugh, Polyhedra: A Visual Approach, University of California Press, Berkeley, 1976.

[27] Venetis, J. An analytic exact form of the unit step function. Mathematics and Statistics, Vol. 2 No.7, 2014, pp. 235-237.

[28] J. Venetis, Analytic Exact Forms of Heaviside and Dirac Delta Function, Advances in Dynamical Systems and Applications, Vol. 15, No. 1, 2020, pp. 115-121

[29] M. Kyle Murphy, Explicit forms of discontinuous functions, The Dirac Delta, and Irreducible Forms, Preprint. https://www.academia.edu/11704122/Explicit_ Forms_of_Discontinuous_Functions_the_Dirac _Delta_and_Irreducible_Forms.

Author Contributions:

John Venetis carried out the simulation and all mathematical derivations and wrote the paper throughout

\section{Sources of funding for research} presented in a scientific article or scientific article itself

This work is totally unfunded

\section{Creative Commons Attribution \\ License 4.0 (Attribution 4.0 \\ International , CC BY 4.0)}

This article is published under the terms of the Creative Commons Attribution License 4.0 https://creativecommons.org/licenses/by/4.0/deed.en US 